\title{
Was Newton right? A search for non-Newtonian behavior of weak-field gravity
}

\author{
Paul Boynton ${ }^{1}$, Michael Moore ${ }^{1}$, Riley Newman ${ }^{2}$, Eric Berg ${ }^{2}$, Ricco Bonicalzi ${ }^{1}$ and Keven McKenney ${ }^{1}$ \\ ${ }^{1}$ Department of Physics, University of Washington, Seattle Washington, USA \\ ${ }^{2}$ Department of Physics, University of California Irvine, Irvine, California, USA
}

\begin{abstract}
Empirical tests of Einstein's metric theory of gravitation, even in the nonrelativistic, weak-field limit, could play an important role in judging theory-driven extensions of the current Standard Model of fundamental interactions. Guided by Galileo's work and his own experiments, Newton formulated a theory of gravity in which the force of attraction between two bodies is independent of composition and proportional to the inertia of each, thereby transparently satisfying Galileo's empirically informed conjecture regarding the Universality of Free Fall. Similarly, Einstein honored the manifest success of Newton's theory by assuring that the linearized equations of GTR matched the Newtonian formalism under "classical" conditions. Each of these steps, however, was explicitly an approximation raised to the status of principle. Perhaps, at some level, Newtonian gravity does not accurately describe the physical interaction between uncharged, unmagnetized, macroscopic bits of ordinary matter. What if Newton were wrong? Detecting any significant deviation from Newtonian behavior, no matter how small, could provide new insights and possibly reveal new physics. In the context of physics as an empirical science, for us this yet unanswered question constitutes sufficient motivation to attempt precision measurements of the kind described here. In this paper we report the current status of a project to search for violation of the Newtonian inverse square law of gravity.
\end{abstract}

\section{Introduction}

In the context of "classical behavior," are the manifestations of gravity fully Newtonian? NonNewtonian potentials exhibit the general property that $\nabla^{2} V \neq 0$ in a source-free region. We choose to represent this condition as

$$
V=V_{\text {gravity }}+V_{\text {Yukawa }}=-\frac{G m}{r}\left(1+\alpha \exp \left(-\frac{r}{\lambda}\right)\right)
$$

where $\lambda$ is the range, and $\alpha$ the ratio of the strength of a putative inverse-square-law-violating (ISLV) interaction to the strength of ordinary Newtonian gravity. The basic experimental design we describe below is founded on a method to detect specifically the existence of a non-Newtonian potential much weaker than gravity, through the unique signature of its non-zero Laplacian, thereby indicating ISLV [1]. Our approach involves a cryogenically cooled torsion pendulum [2] undergoing large-amplitude

This is an Open Access article distributed under the terms of the Creative Commons Attribution License 4.0, which permits unrestricted use, distribution, and reproduction in any medium, provided the original work is properly cited. 
oscillations in proximity to a laboratory-scale source mass. Both pendulum and source mass are carefully configured to exaggerate sensitivity to this specific evidence for non-Newtonian behavior while maintaining insensitivity to Newtonian gravity coupling and electromagnetic interactions. Suppression of these latter effects must be adequate to assure that ISLV detection (or an empirical upper limit) is not biased by extraneous sources of systematic error that would mimic the ISLV signature. Our design goal has been either to detect or to place a $1-\sigma$ upper limit of $\sim 2 \times 10^{-5}$ on $\alpha$ for an interaction range around $12 \mathrm{~cm} \mathrm{[3].} \mathrm{As} \mathrm{indicated} \mathrm{by} \mathrm{the} \mathrm{blue} \mathrm{curve} \mathrm{in} \mathrm{Figure} \mathrm{1,} \mathrm{this} \mathrm{limit} \mathrm{is} \mathrm{roughly}$ an order of magnitude lower than published limits at neighboring ranges between $100 \mu \mathrm{m}$ and $10 \mathrm{~m}$.

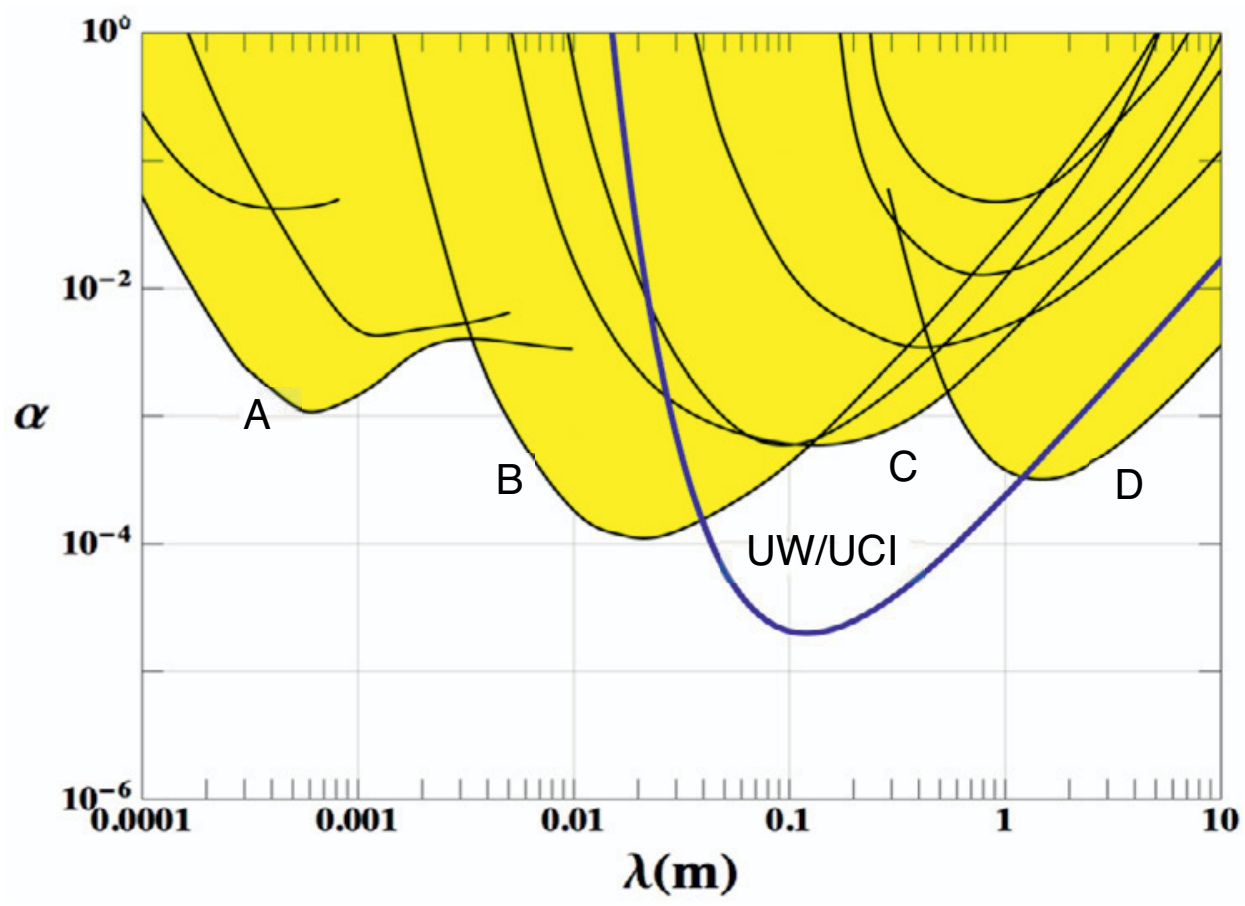

Figure 1. ISLV exclusion plot showing the status of $1-\sigma$ upper limits on positive $\alpha$ values for $\lambda$ between $100 \mu \mathrm{m}$ and $10 \mathrm{~m}$, established by existing, most stringent measurements: A Eot-Wash [4], B Irvine [5], C Irvine [6], and D Maryland [7], with the projected constraint for this project. See also [8].

This project is a collaboration between research groups at the University of California Irvine and the University of Washington in Seattle. Together we have devised novel means to reduce gravitational systematic errors (which historically have limited the sensitivity of macroscopic-range ISLV searches), and to improve measurement precision. The UCI group made a singular contribution to this project by providing a fully developed torsion pendulum cryostat with associated control and data acquisition systems that allowed us to exploit cryogenic operating conditions [2]. Another asset to this work has been the ultra-low-vibration environment of our underground laboratory, the Battelle Gravitation Physics Laboratory (BGPL), established through the joint sponsorship of the National Science Foundation and the Pacific Northwest National Laboratory (PNNL) in a former Nike missile bunker on a remote portion of the DOE-Hanford site in eastern Washington State. At that location we pursued this laboratory-scale search for non-Newtonian violations of the foundations of GTR for the past decade. In the spirit of physics as an empirical science, we have long toiled under the conviction that the only motivation experimentalists require is to measure, rather than to speculate - ironically, as most famously urged by Sir Isaac Newton PRS. 
We are currently analyzing the data acquired during this UW/UCI project. Because this analysis is being conducted as a blind exercise, only preliminary, empirical performance estimates are available at this time. No statements in this progress report should be considered even to suggest "final results."

\section{Experimental design}

Central to our measurement strategy is an experimental design that includes four important features to assure that our ISLV test qualifies as a true null experiment - that the absence of a significant ISLV "signal" implies the absence of ISLV down to a threshold determined by statistical and systematic error estimates:

1) Rather than attempting to measure the force between two test bodies as a function of their separation, we monitor an observable directly related to the departure from zero of the source-free Laplacian of the interaction potential between a pendulum and source masssuch a departure, if detected by this experiment and independently verified by others, would constitute the ISLV we seek and suggest physics beyond the Standard Model.

2) Our ISLV test is framed in a context that assures searching specifically for a non-Newtonian force. We employ a practical design and analysis formalism that cleanly, naturally, and unambiguously separates non-Newtonian from Newtonian interactions.

3) We employ empirical techniques to diagnose and verify adequate suppression of possible non-gravitational (mechanical, electrostatic, and magnetostatic) interactions that can mimic the detection of ISLV.

4) By strongly suppressing both the low-multipole-order, Newtonian mass moments of the pendulum and corresponding field moments of the source mass, we assure that important systematic effects that would arise from these Newtonian gravitational interactions are also strongly suppressed. Under these circumstances, they would arise only in second- and higher-order from fabrication and metrology errors. We thereby avoid heroic effort to meet impractical dimensional and homogeneity tolerances in order to achieve a significant gain in sensitivity to ISLV.

There are two additional design features that merit emphasis. One is important to torsion pendulum performance, the other concerns the context of the analysis of experimental data:

5) The amplitude of the thermal equilibrium noise torque driving the pendulum is proportional to $\sqrt{ }\left(\mathrm{k}_{\mathrm{B}} \mathrm{T} / \mathrm{Q}\right)$ and therefore: 1$)$ is reduced by an order of magnitude through operation at $4 \mathrm{~K}$ rather than at ambient temperature, and 2) by an additional half order of magnitude due to reduced torsion fiber energy dissipation under cryogenic conditions, because of the resulting increase in oscillator quality factor, Q. In practice, because of competing noise sources, we received only a partial reduction in overall noise. Aside from this, cryogenic operation also allows much tighter control of fiber temperature and thereby of troublesome low-frequency drift in torsion frequency

6) Our data analysis is conducted as a blind exercise. The experimental result is hidden until agreement - based on properties of the data other than knowledge of the final result among group members that the analysis process is now fixed and the outcome, without modification, is ready for publication.

Graphic renditions of our choice for pendulum and source mass designs, jointly configured to detect the possible presence of a non-zero Laplacian of the source-free interaction potential are depicted in Figure 2. 


\section{The uniquely non-Newtonian interaction signature of ISLV}

As suggested in the caption of Fig. 2, intuition provides little insight to crafting appropriate pendulum and source mass configurations for the purpose at hand. Conveniently, the multipole-expansion scheme is valid for our design, and provides a conceptual framework that guides the selection of appropriate mass distributions for each member of this pair of interacting elements.

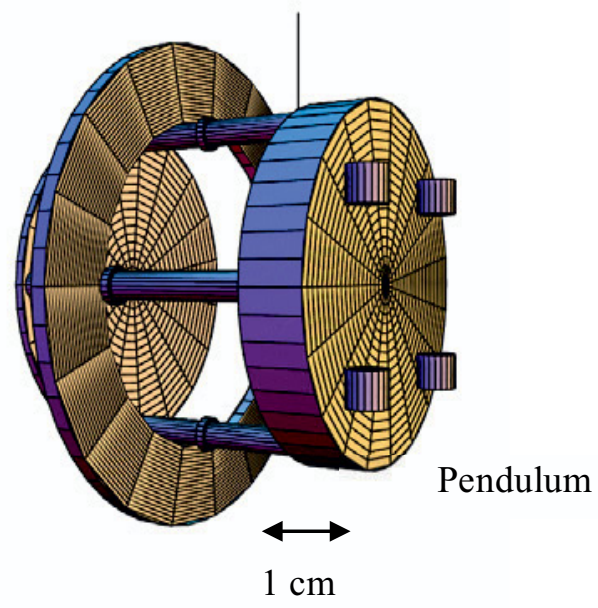

Source Mass

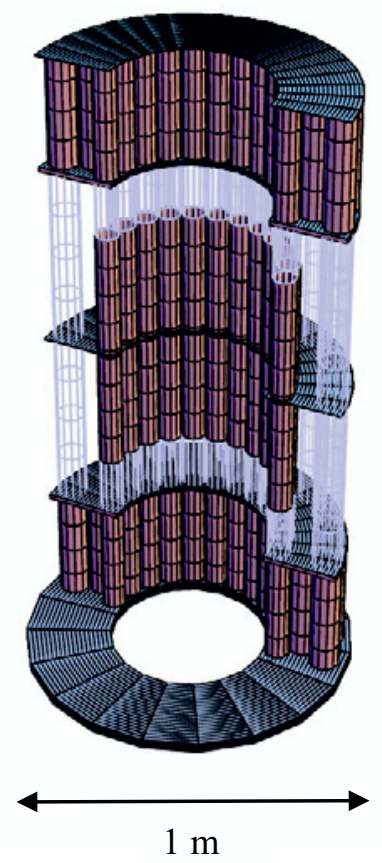

Figure 2. Renditions of source mass and torsion pendulum configurations for detection of a non-Newtonian interaction coupling bits of ordinary matter-why these particular configurations?

Begin by considering the total interaction energy between a pendulum of density $\rho$ and adjacent source mass responsible for potential $V$. Then form the Cartesian Taylor expansion of the interaction energy $U$ about the pendulum center-of-mass:

$$
U=M_{0} V_{0}+\frac{1}{2} M_{x x} V_{x x}+M_{x y} V_{x y}+M_{x z} V_{x z}+\ldots
$$

$$
\text { where } M_{x y}=\iint_{p e n d} \rho_{\text {pend }}(x, y, z) x y d x d y d z
$$

Upon referring to Figure 3, comparison with an expansion in a spherical harmonic basis is revealing:

$$
U=\sum_{n l m} M_{n l m} V_{n l m}, \text { where } \quad M_{h l m}=\iiint_{p e n d} \rho_{\text {pend }}(r, \theta, \varphi) r^{n} Y_{m}^{l} d^{3} r
$$

As illustrated in Figure 3, the scalar, $l=0$, second moment is the lowest-order, non-Newtonian kernel, and as mentioned earlier, by symmetry cannot produce a torque on the pendulum. The first interesting low-order case is the highlighted third moment, $l=1$ coupling. 


\section{“Generalized” Multipole Expansion (as mass moment kernels)}

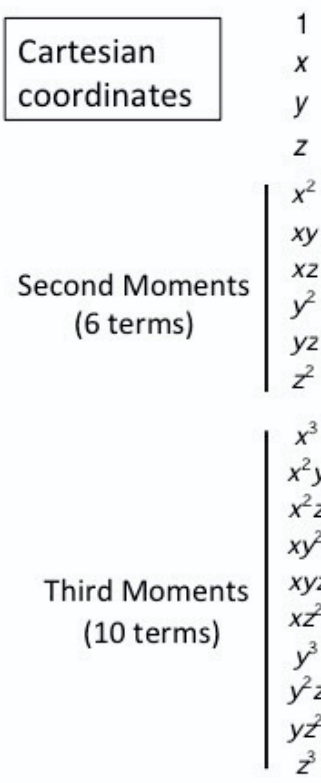

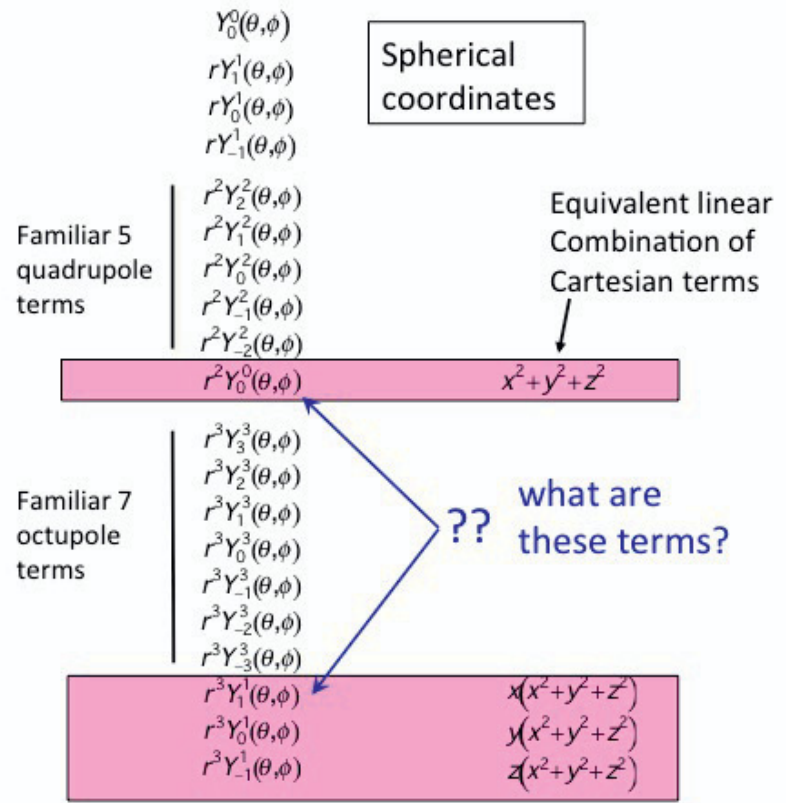

Figure 3. Comparison of Cartesian and spherical coordinate representations of low-order mass-moment kernels in a complete, "generalized" multipole expansion (one that retains non-Newtonian terms). Text book discussions commonly refer only to the five "Newtonian" quadrupole terms, and only to the seven "Newtonian" octupole terms, etc. This is because the one plus three additional terms in the shaded boxes above couple to the Laplacian or its derivatives, which vanish when describing Newtonian interactions. The same pattern holds for kernels of field moments.

\section{Third Moments Are The Key}

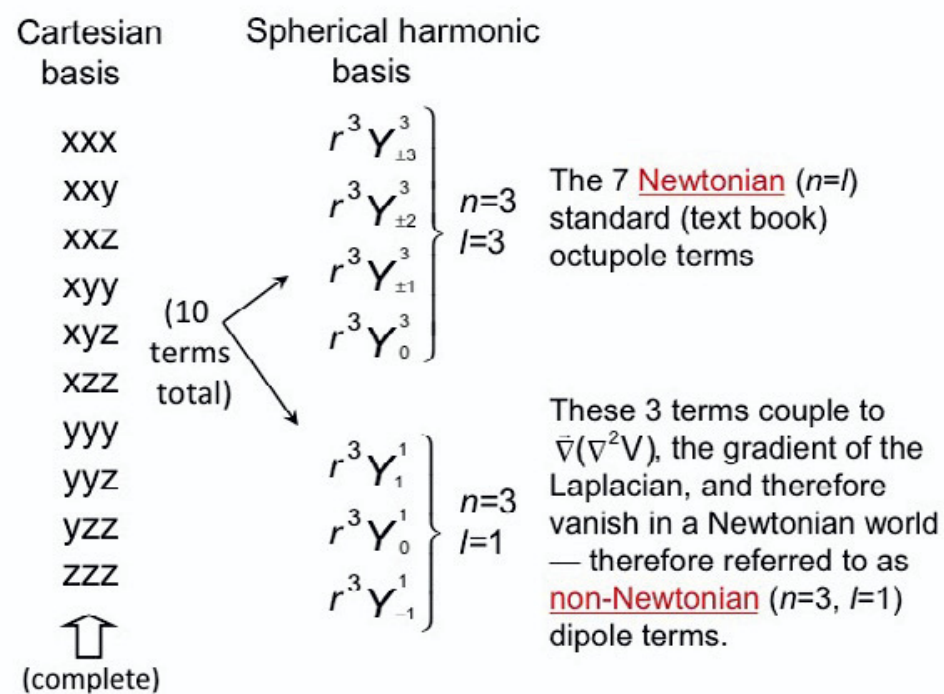

Figure 4. The complete set of ten third-moment terms naturally separate into Newtonian and non-Newtonian classes according the symmetry index $l$. 
This separation of non-Newtonian from Newtonian couplings explicated in Figure 4 is the starting point for our pendulum and source-mass design, based on the following expansion insights:

- The "generalized" or "complete" spherical harmonic representation naturally and uniquely separates non-Newtonian $(l<n)$ from Newtonian $(l=n)$ terms.

- The lowest order, detectable (modulatable) non-Newtonian interaction has specifically an $m=1$ signature

$$
\begin{aligned}
& U_{311}=M_{311} V_{311} \\
& M_{311}=\int \rho r^{3} Y_{1}^{1} d^{3} r \propto \iiint \rho x\left(x^{2}+y^{2}+z^{2}\right) d x d y d z \\
& V_{311}=\frac{\partial}{\partial x} \nabla^{2} V \quad \begin{array}{l}
\begin{array}{l}
\text { by symmetry, } M_{311} \text { clearly couples to the } \\
\text { horizontal gradient of the corresponding }
\end{array} \\
\begin{array}{l}
\text { Laplacian - a distinctly non-Newtonian, } \\
\text { dipole interaction) }
\end{array}
\end{array}
\end{aligned}
$$

- Field moments characteristically decrease by a factor of $\mathrm{R} \propto \mathrm{r}_{\text {pend }} / \mathrm{r}_{\text {source }}$ for each unit increase in $n$ (where $\mathrm{r}$ denotes an effective radius). For our design $\mathrm{R} \approx 0.1$, validating adoption of the multipole expansion, and assuring suppression of higher-order, $m=1$ Newtonian systematic errors, therefore playing a crucial role in suppressing this generally dominant source of systematic error in ISLV experiments.

These insights naturally lead to a design in which the pendulum is configured to exaggerate the nonNewtonian $M_{311}$ mass moment as well as the corresponding $V_{311}$ field moment of the source mass. Exaggerating these does the same for $U_{311}$, which is proportional to the ISLV "signal," the amplitude of torsion period modulation as the source mass rotates.

\section{Fabrication of pendulum and source mass}

The ISLV pendulum displayed in Figure 5 was fabricated at UC Irvine from precisely manufactured fused silica components having $6 \mu \mathrm{m}$ rms dimensional tolerance and flat to a few fringes. The mass is $240 \mathrm{~g}$ and has characteristic linear size of approximately $8 \mathrm{~cm}$. The bare silica elements are bonded together using a room-temperature hydroxide catalysis technique that locally dissolves the contacting surfaces of the fused silica parts, fusing them together with a siloxane bond and liberating water in the process [9]. This technique facilitates precise component positioning while bonding with minimal added mass. The integrity of such bonds survive under repeated cooling cycles to $4 \mathrm{~K}$ when placed in the cryostat. In operation, the pendulum is suspended by a $\mathrm{BeCu}$ fiber, which is stressed to about $60 \%$ of its tensile strength. The pendulum is thoroughly gold coated by an aqueous chemical immersion process, and the fiber electrically grounded to suppress electrostatic torques - the minimum detectable signal energy for $\mathrm{S} / \mathrm{N}=1$ is only on the order of tens of $\mathrm{eV}$.

The components of the ISLV source mass (hereafter SM) shown in Figure 6 were fabricated at the University of Washington. The structure is reassembled by stacking several hundred precisionmachined solid stainless steel cylinders ( $6 \mu \mathrm{m}$ dimensional tolerance) with a mass totaling about 1500 $\mathrm{kg}$. Thin-wall stainless steel tubes (shown in phantom in Figure 2, and visually distinguishable in the first photo of Figure 6) provide structural spacers that support the central and upper portions of the stack. 


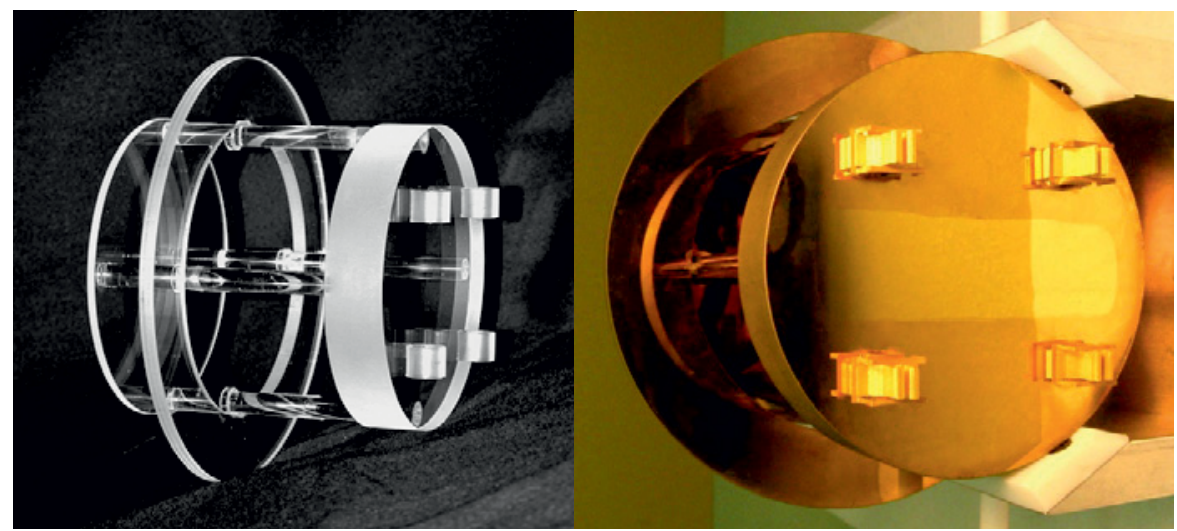

$M_{311} \rightarrow$ large

$\left.\begin{array}{l}M_{11 m}=0 \\ M_{22 m}=0\end{array}\right\}$ for

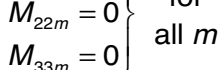

$M_{441}=0$

$M_{551}=0$

$M_{661}=0$

$M_{881}=0$

Figure 5. On left, fused silica ISLV pendulum shown in operating orientation prior to gold coating. CMM verified machining and assembly errors $<6 \mu \mathrm{m}$. On right, view of four, fixed mass, vertical-displacement, trim masses adjusted to suppress 2,1 and 3,1 $(l, m)$ Newtonian mass moments. Reduction of residual moments is diagnosed with exaggerated $V_{221}$ and $V_{331} \mathrm{SM}$ configurations. The solution-deposited, full-coverage, gold coating (silane chemistry bonded) was achieved as a substantial development project completed with excellent results by UW undergraduate, Alan Kalet. Excepted was the optical-quality mirror surface, visible around mid-altitude on the face of the thick disk, which is a vacuum-evaporated gold surface.
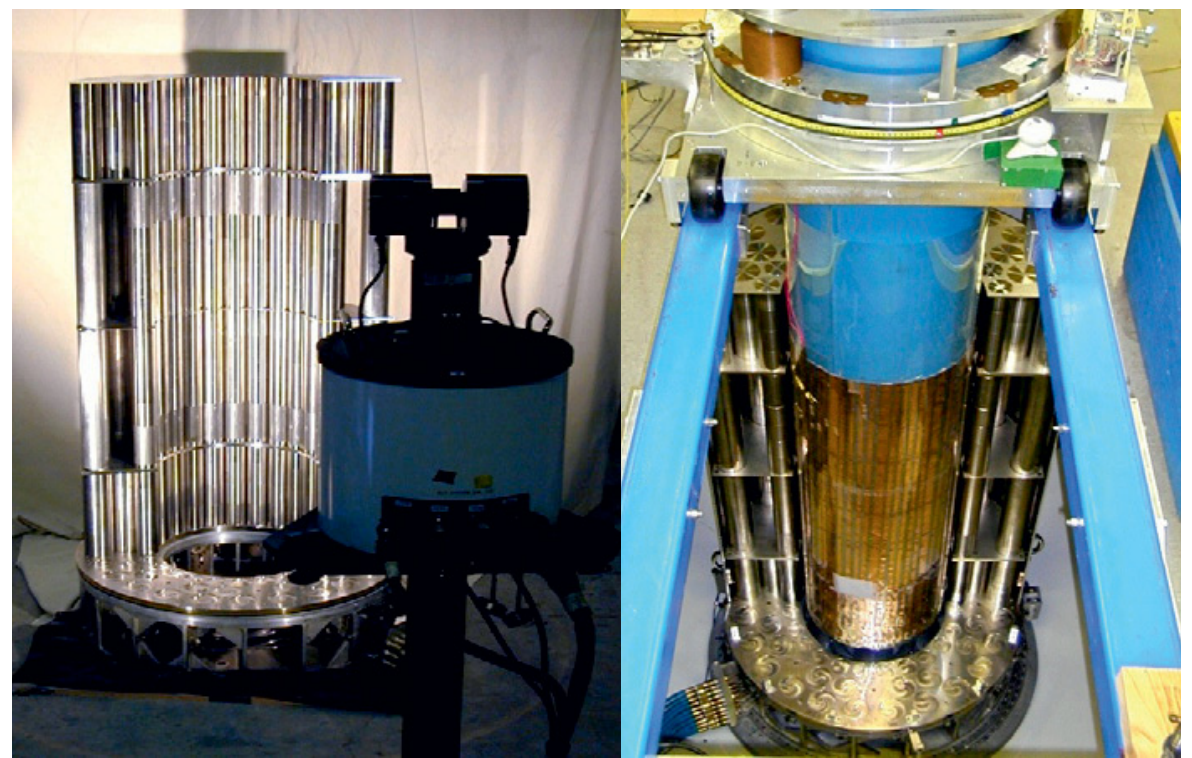

$V_{311} \rightarrow$ large

$V_{22 m}=0 \begin{gathered}\text { for } \\ \text { all } m\end{gathered}$

$V_{331}=0$

$V_{441}=0$

$V_{551}=0$

$V_{661}=0$

$V_{771}=0$

Figure 6. On left, one of several test assemblies of SM. Thin-wall stainless steel tubes (shown phantom in Figure 2, and subtly visible here) provide structural spacers and serve to support the central and top portions of the stack. The 3-D laser ranging CMM that guides the stacking of cylinders is in the foreground. On the right, SM is shown in place, snuggling dewar that cryogenically houses the ISLV pendulum. The pneumatic hoses visible at lower left route $50 \mathrm{psi}$ air to that half of the air bearing directly below the $1500 \mathrm{~kg}$ asymmetric load while the SM advances briefly by $2 \pi / 7$ radians every half hour.

The segmentation of the SM into many solid stainless-steel cylinders would have allowed us to measure and correct for tiny mass differences resulting from machining tolerances and density variations, but mass differences were found to be negligible. Even slight differences in cylinder 
height, however, could accumulate to significant discrepancies when stacking cylinders vertically. This effect was controlled by mixing and matching cylinders according to height differences of a few microns.

As explained in the next section, to limit gravitational systematic errors stringently, the SM design eliminates low-order Newtonian field moments so that such terms arise only from small, statistical departures from design due to cylinder machining and placement errors. This "smallness" requirement was met partly by $6 \mu \mathrm{m}$ cylinder machining tolerances, but primarily by restricting 3-D placement errors to $50 \mu \mathrm{m}$ relative to the design positions.

Finally, 2-D lateral adjustments for each cylinder must be made while stacking to meet the placement-error criterion. Alternate ends of cylinders have shallow male/female mating interfaces, and a $15 \mu \mathrm{m}$ lateral clearance in the interface allows for corrections in the placement of each cylinder as the stack is assembled. The precise placement of cylinders was guided in real time during assembly (and spot checks made during disassembly) with indispensible, 3-D, laser-ranging coordinate measuring equipment left on indefinite loan to us by the Boeing Company in Seattle. The optical head of this fully automated (including preprogrammed target acquisition), state-of-the-art device is visible in the first image of Fig. 6.

\section{Null-experiment strategy}

It is important that the pendulum and SM designs exaggerate the non-Newtonian signal, but equally crucial that systematic effects (particularly those that mimic ISLV) do not dominate the expected measurement error. Thus a second constraint: that the experimental design eliminates low-order, $m=1$, Newtonian gravity couplings between pendulum and SM. Only then can this project qualify as a null experiment - that the absence of a significant ISLV "signal" implies the absence of ISLV down to a threshold determined by statistical and systematic error estimates. In this spirit, we have employed a third constraint: we rule out post-data-acquisition corrections applied during data analysis in order to mitigate systematic effects.

Our source-mass design specifically required that all $m=1$ Newtonian field moments be zero for $l<9$. The even $l, m=1$ terms are naturally zero because of source-mass-configuration inversion symmetry about the horizontal mid-plane. In addition, the design included four degrees of freedom chosen to null the $331,551,771$, and 220 moments. The 220 moment is eliminated to render the SM insensitive to small tilts about a horizontal axis that would generate a 221 moment. The somewhat more complicated design scheme used to null low-order, Newtonian, pendulum mass moments for $k 7$ is discussed in reference 8. All moments nulled by design are listed in Figs. 5 and 6, adjacent to images of the physical realizations of pendulum and SM.

Setting these low-order Newtonian moments to zero in the design assures that only residual Newtonian couplings arise through slight but inevitable fabrication deviations from the null design. As a result, this null-by-design stratagem ensures that potential ISLV-mimicking contributions arise only as second-order (or higher) in the already small fabrication "errors." This suppression of systematic error contributions for lower $l$ values, is continued for higher $l$ values by having chosen $\mathrm{R}<<1$ as explained in section 3 .

Driven by concern that additional measures might be required to achieve the high-precision measurement goals of this project, we estimated how effective we could expect this null-designremedy for systematic-error reduction scheme to be. Upon completion of basic pendulum and SM designs required by the aforementioned constraints on non-Newtonian and Newtonian couplings, and prior to finalizing those designs, we initiated an empirical study to characterize quantitatively the inevitable deviations from these specific designs that would result from best-practice machining and placement techniques, as well as from the effect of material density inhomogeneity. Measured fabrication errors from test parts and assembly trials were compiled, but not treated simply as statistical samples. Instead, "worst-case" estimates for mass and field moments were calculated based on these rms errors by applying them to our basic pendulum and SM models as independent, 3-D displacements of each design-component surface, chosen to produce moment-by-moment the 
maximum contribution when integrating over these exaggerated-error models of the proposed pendulum and SM geometries. These exaggerated, residual Newtonian moments were then used to form conservative upper bounds on equivalent contributions to $\alpha$, and are displayed in the second column of Table 1 .

Table 1. The second column shows the low-multipole-order Newtoniangravitation, $m=1$, systematic-error contributions to $\alpha$ estimated from the worst-case fabrication errors of pendulum and SM. The 661 and 881 multipoles and those higher than 991 contribute $<10^{-8}$. The third column displays the $l=2,3$ contributions after incorporating and adjusting trim masses in both pendulum and SM designs. The $l=4$ contribution was measured but not trimmed.

$\begin{array}{ccc}\begin{array}{c}\text { Multipole } \\ n, l, m\end{array} & \begin{array}{c}\text { Equivalent } \alpha \\ \text { (worst-case) }\end{array} & \begin{array}{c}\text { Equivalent } \alpha \\ \text { (final values) }\end{array} \\$\cline { 1 - 2 } $\left.2,2,1 & 8.0 \times 10^{-5} & 4.6 \times 10^{-7} \\ 3,3,1 & 1.3 \times 10^{-5} & 8.6 \times 10^{-7} \\ 4,4,1 & 6.0 \times 10^{-6} & 4.1 \times 10^{-6}\end{array}\right\}$ measured

These worst-case entries in Table 1 made it clear that further reduction in the magnitudes of $l=2,3$ Newtonian moments would be required for both pendulum and SM as well as means to measure empirically their contamination of the data and to make appropriate adjustments. Measurements were later undertaken with specially configured pendulum and SM configurations [3]. We then adjusted strategically positioned trim masses to null iteratively the residual mass and field moments for $l=2,3$. The $l=4$ term was measured also, but subsequently left untrimmed as too labor intensive a task given other priorities.

The four trim masses that were included in the pendulum design are visible in Fig. 5. These are fixed mass, but independently adjustable in altitude (as pictured), to provide four adjustable degrees of freedom to null the $l=2,3$ mass moments. A similar, larger mass set were attached on the backside of the SM (not visible in Figure 6) to null the $l=2,3$ field moments. Gravitational design modeling for both pendulum and SM took into account the distribution of all these mass elements.

The more straightforward experimental design choices, diagnostic measurements and empirical tests to quantify the suppression of other possible sources of systematic error (electrostatic, magnetostatic, optical, electronic, mechanical, etc.) are not reviewed in this report. In Table 2, we provide the results of a tentative, preliminary survey of noteworthy systematic errors. None of which is problematic. On the other hand, some design issues were quite complex.

For example, there are several channels through which higher $m$ mass and field moments may be aliased to produce $m=1$ contributions. The identification and suppression of several such mechanisms would require a detailed presentation and are also not covered in this brief report, which focuses on basic design issues and their resolution.

Table 2. We have a substantial archive of systematic error test data, which has yet to be studied in detail. The numbers in this table may be revised prior to publication of final results of this ISLV project.

\begin{tabular}{|c|c|}
\hline \multicolumn{2}{|c|}{ Tentative, first-pass, upper limits on } \\
\hline \multicolumn{2}{|c|}{ systematic errors for this experiment } \\
\hline Systematic error source & Contribution to $|\alpha|$ \\
\hline Magnetic field & $|\alpha|<3 \times 10^{-6}$ \\
\hline Instrument tilt & $|\alpha|<2 \times 10^{-6}$ \\
\hline Fiber temperature & $|\alpha|<3 \times 10^{-6}$ \\
\hline Newtonian gravity & $|\alpha|<5 \times 10^{-6}$ \\
\hline Quadrature sum & $|\alpha|<8 \times 10^{-6}$ \\
\hline
\end{tabular}




\section{Experimental method}

Given adequate suppression of ISLV-mimicking systematic effects, at some level a putative coupling between the exaggerated horizontal gradient of a non-Newtonian potential associated with the SM and the exaggerated, non-Newtonian, $m=1$ mass moment of the pendulum could dominate. Throughout the duration of this experiment, the orientation of the equilibrium position of the pendulum is fixed in laboratory coordinates as it undergoes large-amplitude torsion oscillations of $\sim 1.7 \mathrm{rad}$. This amplitude was chosen to be near $1.84 \mathrm{rad}$, which maximizes $\mathrm{S} / \mathrm{N}$ ratio for measurement of the torsion period [10], while satisfying another constraint involving an arcane systematic error specific to this experiment. As the SM rotates about the axis defined by the torsion fiber, the $m=1$ variation in pendulum potential energy associated with this non-Newtonian interaction would manifest as a sinusoidal $(m=1)$ modulation of the torsion oscillation period - the ISLV signature. Given the measured noise performance of our experiment, and for a 60 -day averaging interval, $\mathrm{S} / \mathrm{N}_{\text {statistical }}=1$ corresponds to approximately $4 \mathrm{~ns}$ rms period modulation, and is equivalent to $\alpha_{\mathrm{rms}}=2 \times 10^{-5}$, nearly five orders of magnitude weaker than Newtonian gravity.

To enable rotation of a proximate, $1500 \mathrm{~kg} \mathrm{SM}$ with minimal perturbation of the motion of the pendulum and axial stress of the torsion fiber, the SM is assembled on a 1m-diameter air bearing that facilitates an ultra-low-vibration, step-wise rotation of the SM about the axis of the torsion fiber in seven $2 \pi / 7$ increments over repeated 3.5-hour cycles. During the 30-minute data-acquisition intervals while the period of the pendulum is measured and recorded for each of these seven, stationary sourcemass orientations, the air bearing is shut off and the SM is supported by physical contact with the bearing base. The change in altitude of the bearing load is roughly $50 \mu \mathrm{m}$ between air-on and air-off states, and the gradual pneumatic transition requires several seconds. The bundle of blue pneumatic supply lines that connect the air bearing to the electro-mechanical manifold that controls bearing activation is visible in the right-hand image in Figure 6. In this image (looking south and downward) note that the source-mass air bearing rests on the floor of a lower level room located about 2 meters below the main floor of the laboratory space viewed in Figure 7.

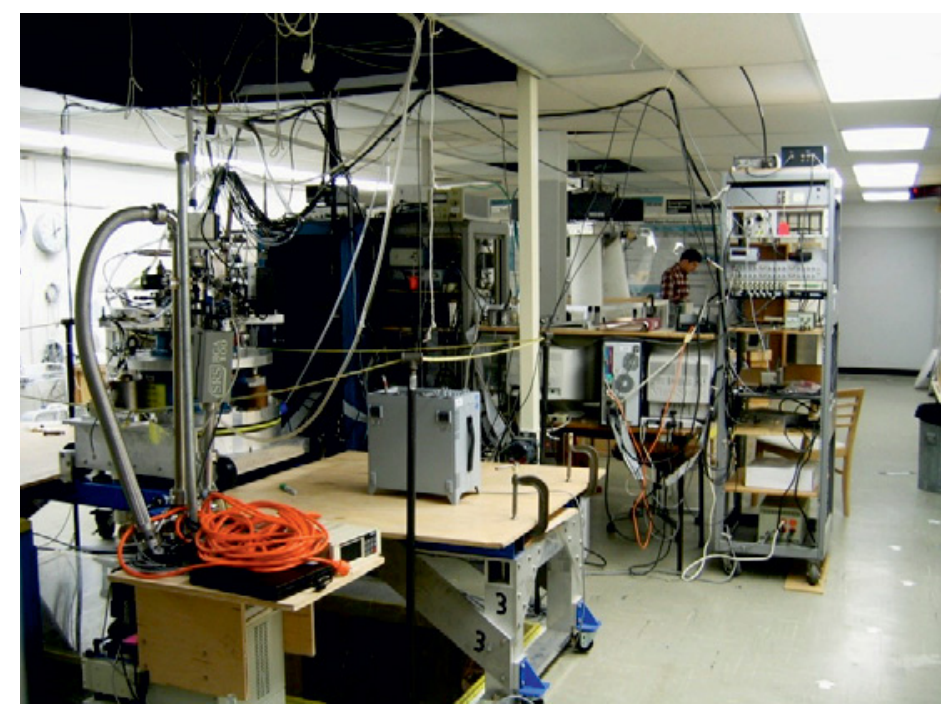

Figure 7. BGPL primary laboratory area on main floor. The dark area at the bottom left is the opening to that space below. View to east.

This lower level room is about $3 \mathrm{~m}$ wide and extends $\sim 20 \mathrm{~m}$ along the E-W mid-line of the main floor. The details of this lower-level floor and its 2-m vertical walls that join the main floor, are important only insofar as they comprise the rigid, massive support for the entire ISLV apparatus. These concrete 
structures are $40 \mathrm{~cm}$-thick, laced with 1" steel rebar, poured on grade, and cured for nearly 60 years the fortunate legacy of occupying a decommissioned Nike missile bunker properly over-built by the US Army Corps of Engineers.

The SM assembly rests on the lower floor, but the dewar containing the torsion pendulum cryostat is supported by the pair of blue, 6" steel box beams backed up by 12" aluminum I-beams that together span the 3 meters between the tops of the facing, heavy walls. These structural elements are visible in Figure 8 , in which the top of the SM is also seen through the opening in the main floor at the bottom left in this photo. As the substantial, eccentric load on the air bearing rotates, the support-structure strain transmitted mechanically to the dewar results in a synchronous tilt less than $300 \mu \mathrm{rad}$, which is measured to be a negligible contribution to the systematic error budget.

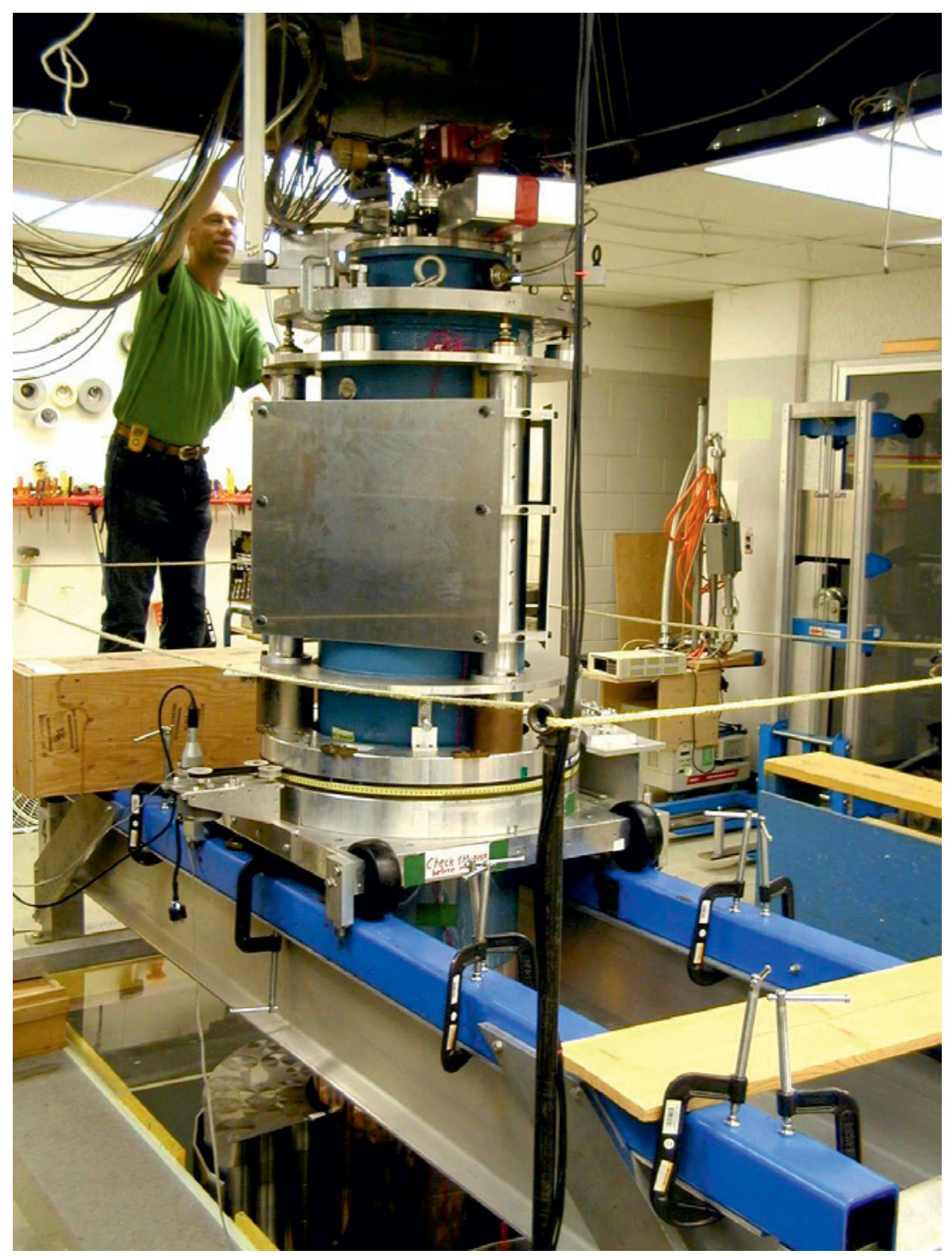

Figure 8. ISLV instrument ready for operation as viewed from main floor. Upper portion of SM is visible at the bottom left. 


\subsection{Dewar and cryostat}

The lower portion of the blue, 100L-capacity LHe dewar that houses the pendulum cryostat is visible in Figure 6, the upper portion that extends into the main lab is shown in Figure 8. As seen on the left in Figure 9, this 6-day-hold-time, superinsulated dewar maintains a $4.2 \mathrm{~K}$ environment for the cryostat vacuum can, which contains the torsion pendulum, its temperature-regulated fiber suspension point, and optical components to measure the torsion period. See Figure 10 as well.

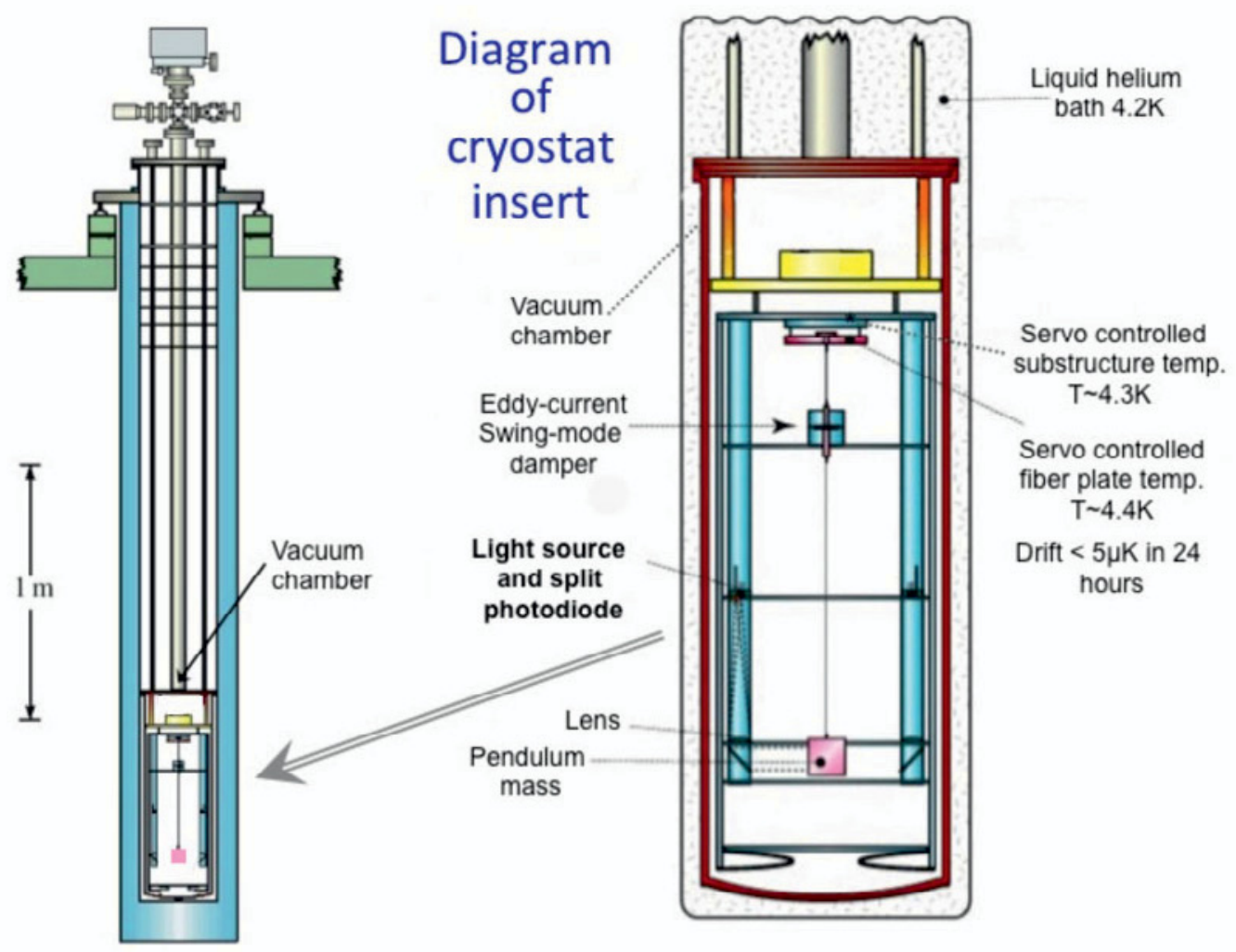

Figure 9. Highly simplified schematic depiction of UCI cryogenic torsion pendulum cryostat showing primary components. The photos in Figure 10 better reveal the essential complexity.

As mentioned briefly in section 2, operation of the torsion pendulum at cryogenic temperature offers several potential advantages:

- Our primary observable, torsion period, depends on fiber temperature through the elastic modulus, but this temperature sensitivity becomes small at $4 \mathrm{~K}$. Consequently, high-stability temperature control is readily achieved.

- Contribution to the total noise budget from thermal-equilibrium-fluctuation noise, whose amplitude scales as $\left(\mathrm{k}_{\mathrm{B}} \mathrm{T} / \mathrm{Q}\right)^{1 / 2}$, becomes negligible. This is due to the reduction in $\mathrm{T}$ and also the $\mathrm{Q}$ of our $\mathrm{CuBe}$ fiber is consequently increased by and order of magnitude.

- The high pendulum Q ensures that data may be accumulated for several days before the need to re-excite oscillation amplitude.

- Highly effective shielding from the changing external magnetic field environment is provided by a layer of super-conducting lead that covers the vacuum can. 
The UCI group developed this cryogenic pendulum instrument, which was successfully used in their measurement of the gravitational constant, G [2]. With some design foresight, no significant modifications were required to accommodate the ISLV pendulum. The dewar/cryostat is mounted on a precision turntable (visible as the black and yellow band girdling the instrument in Figure 8) that allows it to be rotated under computer control to excite or de-excite torsional oscillations to acquire the appropriate oscillation amplitude.

The cryostat vacuum chamber is suspended from the top of the dewar by a 2.5 " diameter, thinwall, stainless-steel pumping tube, plus a smaller tube to bring fiber optic and photodiode cables into the vacuum chamber. A LHe fill tube is also present. The optical fiber brings $850 \mathrm{~nm}$ light from an IR LED to be collimated and reflected from the pendulum mirror surface when rotating through the equilibrium orientation, then refocused on the split photodiode. Auxiliary mirrors mounted in the vacuum chamber provide multiple reflections from the pendulum and produce a total of five beamcrossings of the photodiode associated with each pendulum passage through the equilibrium position. Successive crossings were time-stamped with the output from a $10 \mathrm{MHz}$ GPS-disciplined crystal oscillator, and later used to determine each torsion period.

The temperature of the fiber support point (and thus the fiber) is tightly regulated to less than $1 \mu \mathrm{K}$ drift over each successive interval of 3.5 hours required for the SM to complete one full rotation the time to complete a single, statistically independent estimate of the ISLV "signal."

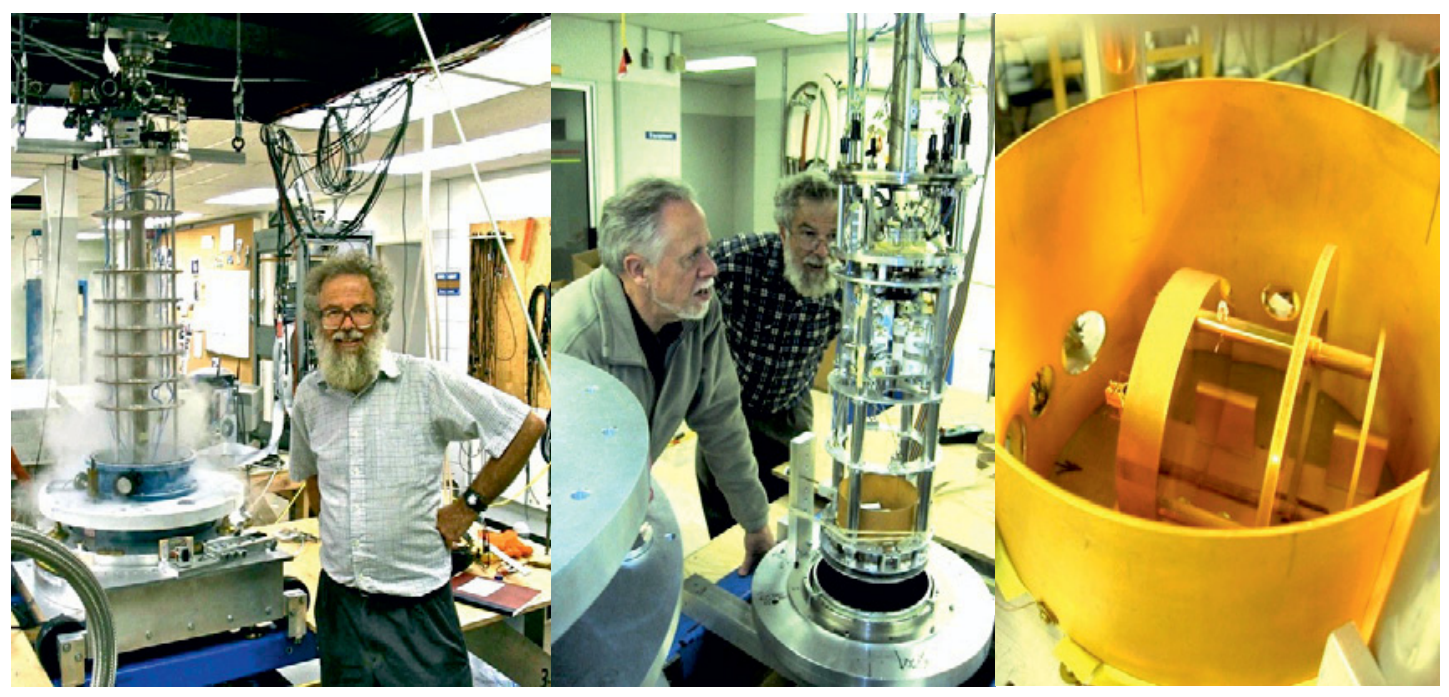

Figure 10. The left image shows a rare occasion on which the cold cryostat was withdrawn for modification. In the center, the vacuum can is removed, revealing the several instrumentation systems within. Most visible is the gold-plated-copper electrostatic shield that surrounds the ISLV pendulum, shown close up in the far right panel.

\subsection{Run protocol}

Prior to each 3-day data acquisition run, after LHe refill, the pendulum oscillation amplitude was pumped up to a standard value and all personnel left the lab. Vacating was essential because the mechanical vibration background resulting from any human presence in our remote, underground laboratory was found to greatly compromised the ambient $\mathrm{S} / \mathrm{N}$ ratio. The data acquisition sequence began automatically 10 minutes later. In the conduct of this experiment, every important aspect of data acquisition and instrument control was fully automated and system status continuously available via internet for remote monitoring and remote intervention if required. 
Operation sequence:

1) Ten split-photodiode "crossings" by IR beam are digitized in each $188 \mathrm{~s}$ torsion period.

2) After ten pendulum oscillation periods ( $\sim 30$ minutes), the SM was advanced by $2 \pi / 7 \mathrm{rad}$.

3) After seven SM moves ( $\sim 3.5$ hours), one SM circuit was completed and the SM rotation sequence continued.

4) Typical run duration was $20 \mathrm{SM}$ circuits ( $~ 3.0$ days), followed either by pumping the torsion amplitude up to the standard, initial value, then starting the next run immediately — or, after the passage of 6 days, by a manual $\sim 100 \mathrm{~L}$ LHe transfer. When post-transfer fiber temperature regulation had been established in $\sim 4$ hours, the torsion amplitude was then pumped up to the standard initial value and the next 3-day run started

This sequence continued with only a few interruptions, 24 hours a day, 7 days a week from late-April to mid-September 2011. See Figures 12 and 13 for images of BGPL entrance and intrepid staff.

We are currently analyzing 19 low-noise runs selected as uniformly consistent with the same, wellstudied noise model. After applying time-domain-filter techniques to suppress low frequency noise, we fit the data in these 19 runs to a function containing harmonic sinusoidal terms with $m=1,3,4,5,6$ that depend on SM position angle and have normalized Bessel function coefficients to accommodate the decline in torsion amplitude during each run. Because our analysis code leaves us intentionally blind to the ISLV signal, only the statistical uncertainty of the $m=1$ fit is accessible.

To provide a glimpse of the character of the data, the upper plot in Figure 11 shows a least-squares fit to a succession of torsion-period measurements (one composite period measure for each SM position) for a portion of a typical run. The lower plot depicts the same data sample showing the individual harmonic components except the $\mathrm{m}=1$ contribution.

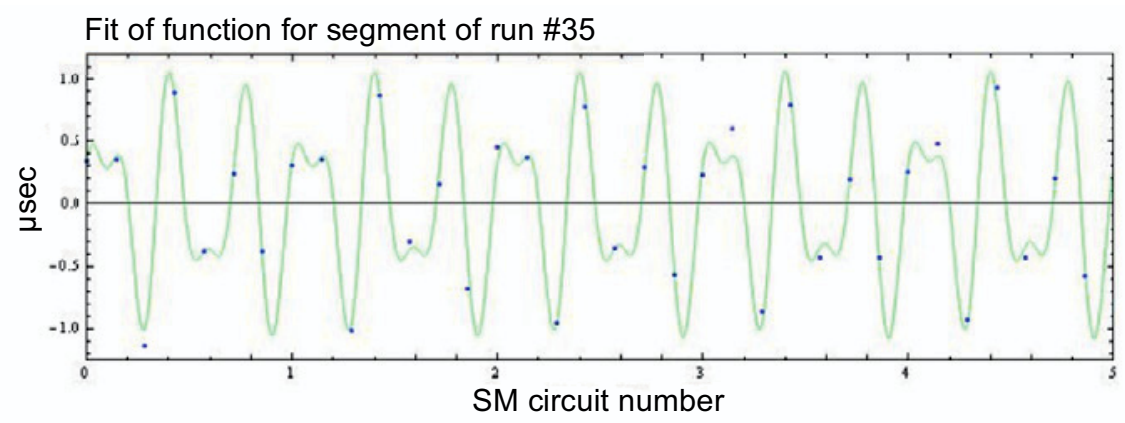

All fit function components except $m=1$ signal channel

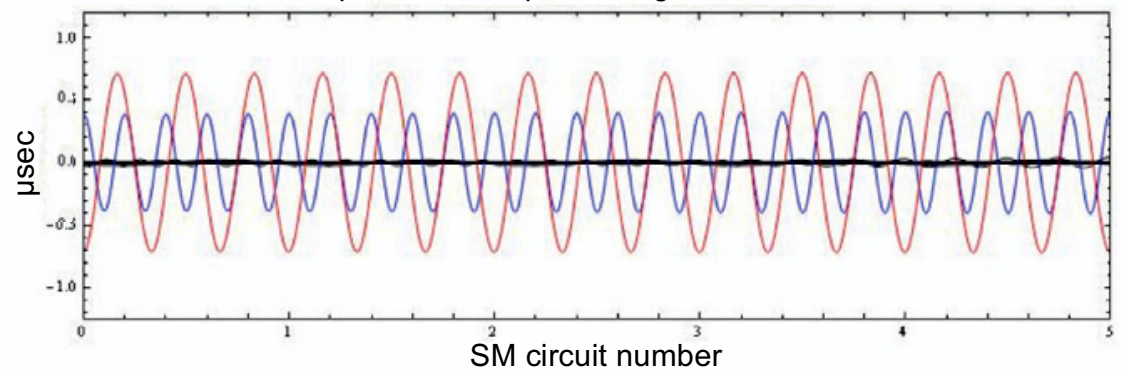

Figure 11. Sample of data showing substantial harmonic content dominated by harmless $m=3$ and $m=5$ Newtonian gravity "signals." 


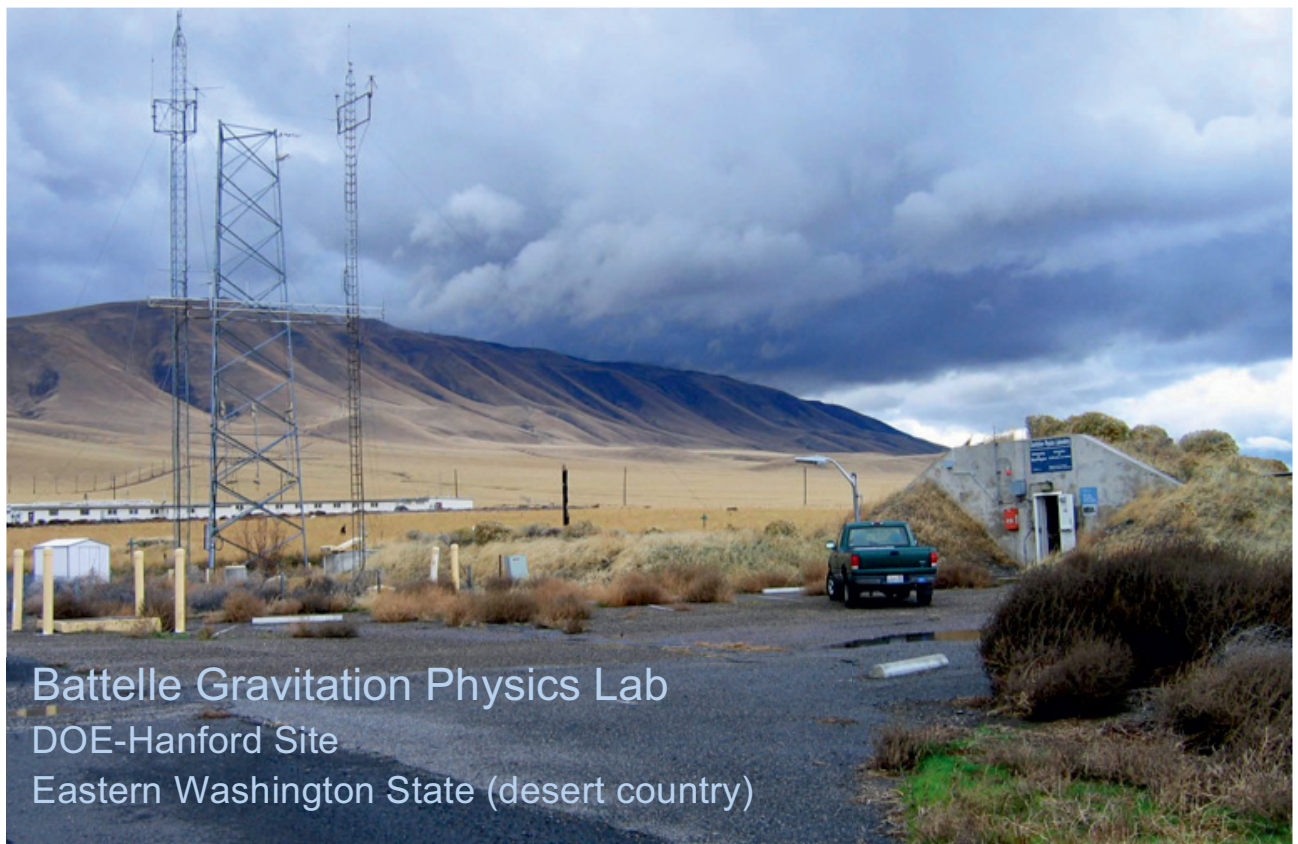

Figure 12. A stormy day at the entry stairs that lead down our remote, underground lab for gravity studies provided by PNNL. Located deep in a US Government restricted access area, approximately $7 \mathrm{~km}$ from a locked security perimeter and the nearest public highway. LIGO lies $12 \mathrm{~km}$ to the northeast. Rattlesnake Mountain rises 700 meters above the desert floor in the background, with no trees.

Figure 13. Major contributors to the successful conduct of this research project. From left, Riley Newman, Keven McKenney, Michael Moore, Paul Boynton, Ricco Bonicalzi, Eric Berg.

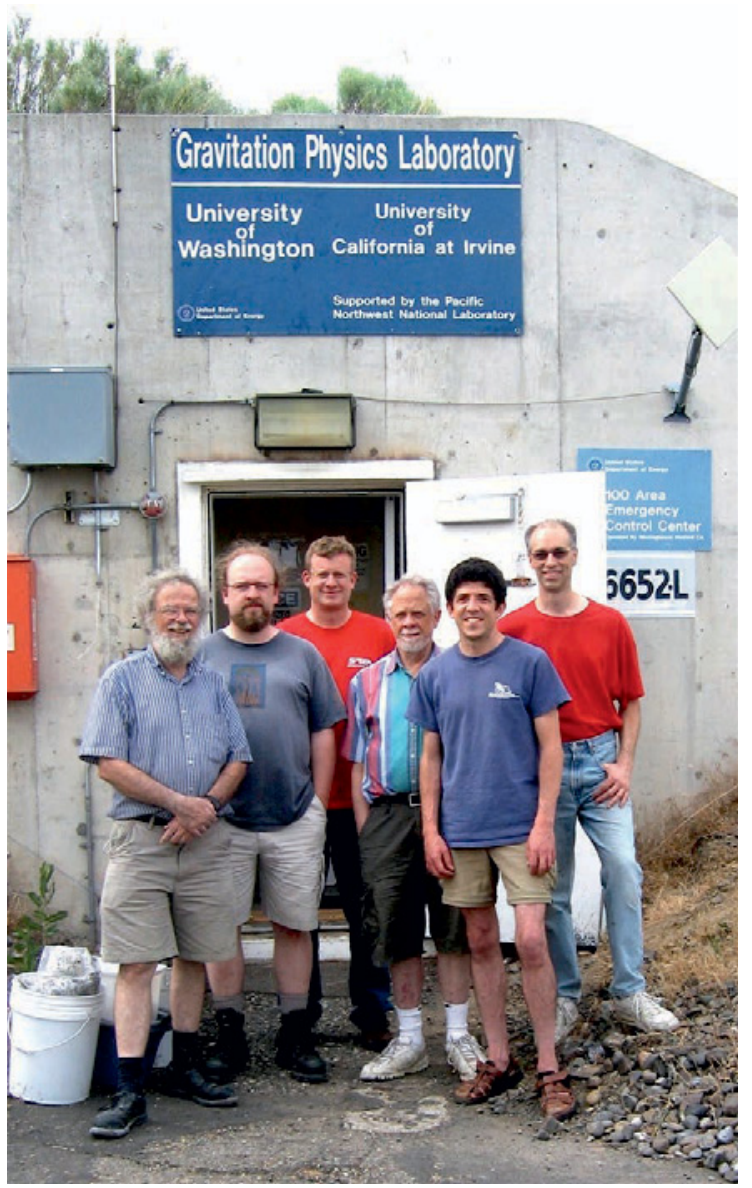




\section{Acknowledgments}

We are grateful for sponsorship by PNNL and The Boeing Company, with special appreciation to Roy Gephart for years of masterfully and productively facilitating our interaction with PNNL, and to Dr. Jonathan Saint Clair for his support by enabling the crucial, long-term loan of a Boeing Laser Tracker CMM. We also deeply appreciate two decades of patient, responsive, sine qua non research funding by the National Science Foundation, most recently through grants PHY-0701923 (UW) and PHY0404514 (UCI).

\section{References}

1. M.W. Moore, A. Boudreau, M. DePue, J. Guthrie, R. Legere, A. Yan, P.E. Boynton, Class. Quantum Grav. 11, A97 (1994)

2. R. Newman, M. Bantel, E. Berg and W. Cross, submitted to Phil.Trans. R. Soc. A (2014)

3. P.E. Boynton, R.M. Bonicalzi, A.M. Kalet, K.J. McKenney, M.W. Moore, J.H. Steffen, E.C. Berg, W.D. Cross, R.D. Newman, R.E. Gephart, New Astronomy Reviews, 51, 334 (2007)

4. D.J. Kapner, T.S. Cook, E.G. Adelberger, J.H. Gundlach, B.R. Heckel, C.D. Hoyle, and H.E. Swanson, Phys. Rev. Lett. 98, 021101 (2007)

5. R. Spero, J.K. Hoskins, R. Newman, J. Pellam, and J. Schultz, Phys. Rev. Lett. 44, 1645 (1980)

6. J.K. Hoskins, R.D. Newman, R. Spero, and J. Schultz, Phys. Rev. D 32, 3084 (1985)

7. M.V. Moody, H.J. Paik, Phys. Rev. Lett. 70, 1195 (1993)

8. R.D. Newman, E.C. Berg, P.E. Boynton, Space Sci Rev 148, 175 (2009)

9. D-H. Gwo, Proc. SPIE 3435, 136, (1998)

10. P.E. Boynton, Class. Quantum Grav. 17, 2319 (2000) 